\title{
Update
}

\section{Role of the histopathologist in assessment of the rejection patterns of renal allo- graft biopsies}

\section{B S Seneviratne}

Renal biopsies on transplanted kidneys are performed to determine whether the compromised graft function is due to rejection, nephrotoxicity caused by immunosuppressive drugs or other causes such as acute tubular necrosis, acute pyelonephritis, and obstruction of the vasculature or due to recurrent glomerular disease.

A renal biopsy, whether it is from a native or a transplant kidney, is an invasive procedure used to obtain a morphological diagnosis and to get prognostic information to guide treatment. Thus, when a patient is "put at risk" by an invasive procedure, the goal should be to collect sufficient amount of tissue to render the best possible diagnosis (1).
Histopathologist plays an important role in identifying the rejection pattern based on the morphological features of the allograft biopsy (2). Initial light microscopic assessment of allograft biopsies can be supplemented by immunohistochemical and immunofluorescence studies to arrive at a definitive diagnosis (3).

\section{Adequacy of renal allograft biopsies}

Recommended method to obtain a satisfactory sample via the percutaneous method is to perform the renal allograft biopsy under ultrasound guidance using a biopsy gun. Biopsy guns have an excellent record of safety in the absence of common contraindications.

Department of Pathology, Faculty of Medical Sciences, University of Sri Jayewardenepura, Gangodawila, Nugegoda, Sri Lanka

Correspondence: M B S Seneviratne, email : bimalka03@yahoo.com 
In a large multicenter study of more than 2,000 protocol biopsies, no patient deaths were reported (4). Similar results were more recently published from a large single center in Germany (5). Ultrasound guidance increases the probability of obtaining tissue from the cortex from 75 percent to more than 90 percent. Guidance by on-site examination with a dissecting microscope increases tissue adequacy to nearly 100 percent.

Open wedge biopsies typically require anesthesia and are not commonly undertaken after transplantation. Wedges are more frequently obtained during organ procurement to assess the suitability for transplantation or at the time of grafting, that is, implantation zero hour biopsies. Surgeons often prefer wedge biopsies since bleeding can be better controlled and sampling generates large tissue fragments.

Before studying the light microscopic features it is important to assess the diagnostic adequacy of renal allograft biopsies. The criteria for assessing the diagnostic adequacy of renal allograft biopsies are given in table 1 .

\section{Table 1. "Chapel Hill" Criteria for assessing the diagnostic adequacy of renal allograft biopsies $(4,5)$}

1. At least two biopsy cores available for standard light microscopic evaluation.

2. $\geq 12$ glomeruli (located in the deep cortex)

3. $\geq 2$ large interlobular arteries/branches of arcuate arteries (with at least 2-3 layers of medial smooth muscle cells)

4. Portion of medulla

\section{Handling of renal allograft biopsies}

Renal allograft biopsies need to be handled accurately in the laboratory. The cores should be divided for light and immunofluorescence (IF) microscopy. Electron microscopic studies are typically limited to cases in which a glomerulonephritis is suspected. The largest tissue portion should always be processed for light microscopic studies. Classically the laboratory should prepare 2-3 $\mu \mathrm{m}$ thick sections for hematoxylin \& eosin (at three levels), periodic acid schiff, trichrome and elastic tissue stains.

Tissue for IF microscopy can be collected by cutting off one half of one (fresh) biopsy core and examining under a dissecting microscope for the presence of glomeruli. The 
longitudinal splitting of the entire biopsy core is strongly discouraged since it can cause severe squeezing artifacts. Renal tissue for IF studies should be frozen in isopentane. Allograft biopsies should be classically stained by IF method for $\mathrm{IgG}, \operatorname{IgA}$, IgM, complement factor C3, fibrinogen, kappa and lambda light chains and the complement degradation product $\mathrm{C} 4 \mathrm{~d}$.

Instead of IF studies, some centres especially in Europe, perform immunohistochemistry on formalin-fixed and paraffin-embedded tissue sections with good results. If tissue is needed for electron microscopy it can be obtained by cutting off small portions of the fresh biopsy cores (approximately $1-2 \mathrm{~mm}$ of cortex). Only very limited tissue samples with approximately three glomeruli are needed for ultra structural analysis.

\section{Rejection}

Rejection episodes can be classified according to different schemes that guide therapeutic decisions. Frequently transplant physicians prefer a simple classification approach that is mainly based on the temporal occurrence of a rejection episode following surgery.

According to this concept "hyperacute" or "accelerated acute" rejection implies a very early event (within hours or days after transplantation), "acute" rejection is diagnosed within days or weeks, and chronic rejection is considered to be a late event occurring months or years after grafting. In hyperacute rejection, fibrin thrombi are present in renal vessels including the glomerular capillaries and peritubular venules (Fig.1). The thrombosis is associated with acute ischaemic injury, focal hemorrhage, infiltration by neutrophils and in most severe cases frank infarcts. Immunofluorescence studies may show linear staining for immunoglobulins and complement along the walls of the capillaries and peritubular venules. Hyperacute rejection is produced by the interaction of circulating antibodies in the recipient with antigens on donor endothelial cells. The antibodies are often related to previous blood transfusions, pregnancies or an earlier transplantation.

Acute rejection may involve the interstitial compartment and/or vessels. When the vascular compartment has been involved it has been termed acute vascular or acute humoral rejection. The most severe changes occur in the small arteries, veins and arterioles (6). There is swelling and vacuolisation of endothelial cells with focal ulceration. This is associated with intimal infiltration by mononuclear cells and changes in the media such as swelling of medial smooth muscle cells. In addition there can be thrombosis. Occasionally a pattern of necrotiz- 
ing vasculitis may be seen. Glomeruli can show endothelial swelling, increased cellularity and occasional thrombosis. In addition there may be interstitial haemorrhages, tubular necrosis and infarctions.

Acute rejection involving the interstitial compartment has been termed acute interstitial or acute cellular rejection (7). Early stages will show oedema and focal infiltration of the interstitium and peritubular capillaries by lymphocytes. As the rejection continues the inflammatory infiltrate becomes diffuse and the lymphocytes will be accompanied by plasma cells, monocytes and macrophages (Fig. 2). Neutrophils may be present but not in abundance.

A characteristic finding of acute interstitial rejection is invasion of tubular epithelial cells by lymphocytes producing a lesion referred to as tubulitis (Fig.3). The intensity of the infiltrate and tubular injury are features used to grade rejection. The infiltrate is more concentrated in the cortex than in the medulla. Glomerular and vascular changes are invariably present but may be mild (8). Although it is convenient for descriptive purposes to consider the two types of acute rejection separately, almost all the cases of acute rejection are really a combination of interstitial and vascular changes.

Chronic rejection is not a distinct entity but rather the end stage of repeated episodes of acute rejection. Chronic rejection once initiated is irreversible. The glomerular lesions consist of ischaemic glomerular capillary collapse, thickening of the capillary walls and segmental and global sclerosis (9). Tubules show atrophy and there is diffuse interstitial scarring. The blood vessels, especially the interlobular and arcuate arteries show severe obliterative fibrointimal proliferation or mucoid widening of the media (Fig.4).

Immunofluorescence studies may occasionally show linear or granular deposition of $\operatorname{IgM}$, IgG and complement components (10). However these general clinical terms are rather imprecise, for example on histological grounds acute and chronic changes are often not sharply separated entities but rather represent an evolving sequence of morphological changes.

A renal allograft biopsy is generally considered to be the gold standard for the evaluation of clinical or subclinical graft injury and for the classification and typing of rejection episodes. Over the past decades several classification systems for the evaluation of the histological features of renal allograft biopsies have been developed. The best known are Banff and CCTT (collaborative clinical trials in transplantation) classification schemes which combine histologi- 


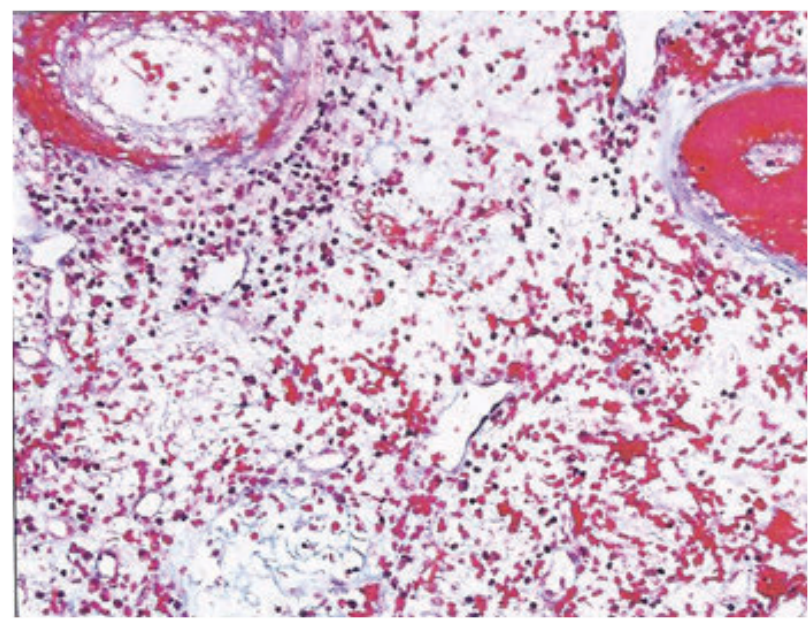

Fig 1: Thrombi in large and small caliber vessels. Interstitum is oedematous and infarcted (H\&E $x$ 400)

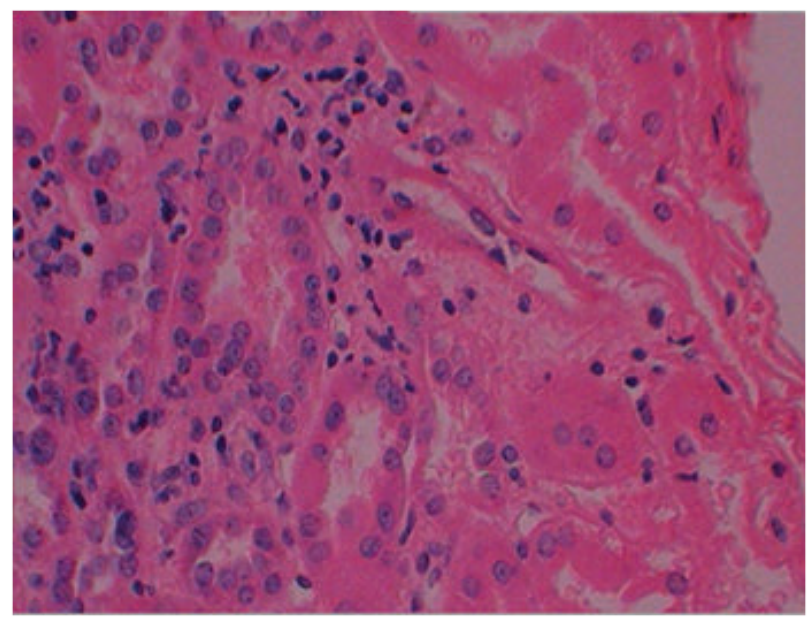

Fig 3: Acute interstitial rejection; the field shows tubulitis (H\&E x 400)

cal, clinical, and pathophysiological parameters of rejection.

Most widely used and accepted is the so called Banff system, introduced in 1993. This system has significantly standardized the classification of renal transplant rejection (11). Over the past years it had undergone several modifica-

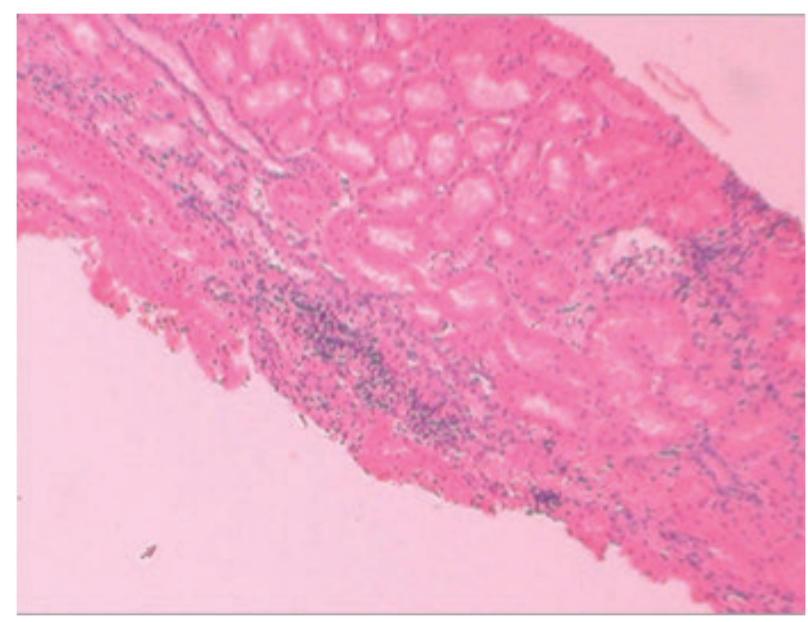

Fig 2: Acute rejection involving the interstitial compartment. Interstitium shows a moderately dense infiltrate of lymphocytes (H\&E x100)

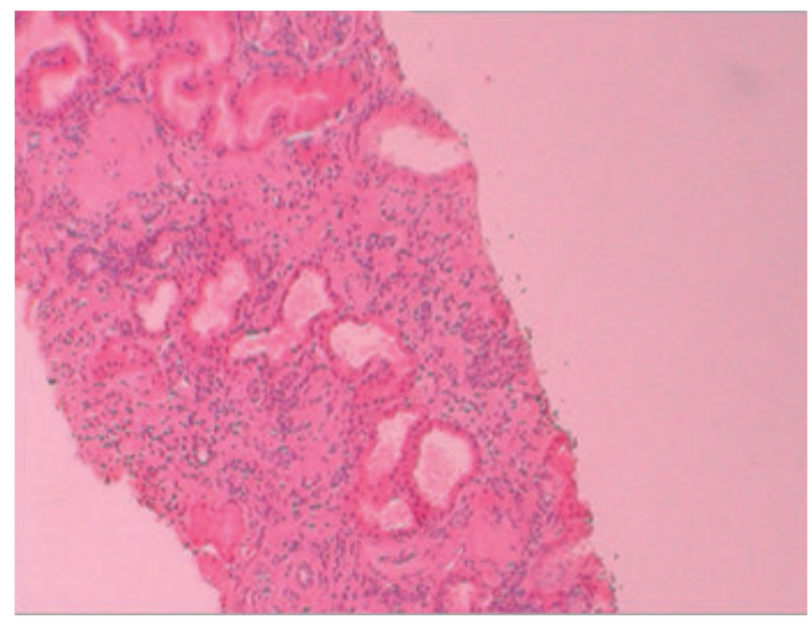

Fig 4: Chronic rejection; there is glomerulosclerosis, chronic inflammation and interstitial scarring (H\&E x100)

tions. (Table 2) In this classification an adequate core biopsy must contain a minimum of 10 glomeruli and at least two arteries; a marginal sample is that with 7 to 10 glomeruli and one artery; and an unsatisfactory biopsy is a core with less than 7 glomeruli or no arteries. 
Table 2 Banff 97 diagnostic categories for renal allograft biopsies (11)

1. Normal

2. Antibody-mediated rejection

A. Immediate (hyperacute)

B. Delayed (accelerated acute)

3. Borderline changes: "Suspicious" for acute rejection

Cases with foci of mild tubulitis (t1- 1 to 4 mononuclear cells/tubular cross section) and interstitial inflammation (i1/mild - $10 \%$ to $25 \%$ of parenchyma affected)

4. Acute/active rejection

Type IA: Cases with significant interstitial inflammation (i2 or i3 $>25 \%$ of parenchyma affected) and foci of moderate tubulitis ( $\mathrm{t} 2>4$ mononuclear cells/tubular cross section or group of 10 tubular cells)

Type IB: Cases with significant interstitial inflammation ( $>25 \%$ of parenchyma affected) and foci of severe tubulitis ( $\mathrm{t} 3>10$ mononuclear cells/tubular cross section or group of 10 tubular cells)

Type IIA: Cases with mild to moderate intimal arteritis (v1)

Type IIB: Cases with severe intimal arteritis comprising $>25 \%$ of the luminal area (v2)

Type III : Cases with "transmural arteritis" and/or arterial fibrinoid change and necrosis of medial smooth muscle cells (v3 with accompanying lymphocytic inflammation)

5. Chronic/ sclerosing allograft nephropathy

Grade I : Mild interstitial fibrosis and tubular atrophy (less than 25\% of the cortical area) without(a) or with(b) specific changes suggesting chronic rejection

Grade II : Moderate interstitial fibrosis and tubular atrophy (26-50\% of cortical area) (a) or (b)

Grade III : Severe interstitial fibrosis and tubular atrophy( $>50 \%$ of cortical area) and tubular loss (a) or (b)

6. Changes unrelated to rejection

(v-vascular, i-interstitial, t-tubular, ptc- peritubular capillaries, mild- 1, moderate - 2, severe - 3) 
The above classification has undergone revisions in 2007 (12), to facilitate the establishment of therapeutic guidelines for clinical management (Table 3)

Table 3- 2007 update of the Banff classification scheme (12)

\begin{tabular}{|l|}
\hline 1. Normal \\
\hline 2. Antibody mediated changes (may coincide with categories $3,4,5$ and 6 ), due to documenta- \\
tion of circulating anti-donor antibody, $\mathrm{C} 4 \mathrm{~d}$ and allograft pathology $\mathrm{C} 4 \mathrm{~d}$ deposition without \\
morphological evidence of active rejection $\mathrm{C} 4 \mathrm{~d}+$, presence of circulating anti-donor antibod- \\
ies, no signs of acute or chronic T-cell mediated rejection or antibody mediated rejection. \\
Acute antibody mediated rejection \\
C4d+, presence of circulating anti-donor antibodies, morphological evidence of acute tissue \\
injury, such as \\
i. ATN like minimal inflammation \\
ii. Capillary and/or glomerular inflammation and/or thromboses \\
iii. Arterial- v3 \\
Chronic active antibody- mediated rejection \\
C4d+, presence of circulating anti-donor antibodies, morphological evidence of chronic tissue \\
injury such as glomerular double contours, peritubular capillary basement membrane multilay- \\
ering, interstitial fibrosis/tubular atrophy and or fibrous intimal thickening in arteries. \\
\hline 3. Borderline changes suspicious for acute T cell-mediated rejection (may coincide with \\
categories 2,5 and 6 ). This category is used when no intimal arteritis is present, but there \\
are foci of tubulitis (t1, t2 or t3) with minor interstitial infiltration (i0 or i1) or interstitial \\
infiltration ( i2, i3) with mild (t1) tubulitis. \\
4. T-cell mediated rejection (may coincide with categories 2 and 5 ) \\
Acute T-cell mediated rejection (type/grade) \\
and foci of moderate tubulitis (t2) \\
\hline
\end{tabular}


IB. Cases with significant interstitial infiltration ( $>25 \%$ of parenchyma affected, i2 or i3) and foci of severe tubulitis ( $\mathrm{t} 3$ )

IIA. Cases with mild to moderate intimal arteritis (v1)

IIB. Cases with severe intimal arteritis comprising $>25 \%$ of the luminal area (v2)

III. Cases with transmural arteritis and/or arterial fibrinoid change and necrosis of medial smooth muscle cells with accompanying lymphocytic inflammation (v3)

Chronic active T-cell mediated rejection, "chronic allograft arteriopathy" (arterial intimal fibrosis with mononuclear cell infiltration, formation of neo-intima)

5. Interstitial fibrosis and tubular atrophy, no evidence of any specific etiology (may include non-specific vascular and glomerular sclerosis, but severity graded by tubulo-interstitial features)

Grade i. Mild interstitial fibrosis and tubular atrophy $(<25 \%$ of cortical area)

ii. Moderate interstitial fibrosis and tubular atrophy (26-50\% of cortical area)

iii. Severe interstitial fibrosis and tubular atrophy/loss ( $>50 \%$ of cortical area)

6. Other : changes not considered to be due to acute or chronic rejection

According to the 2007 update of Banff classification scheme,

-Category 1 is similar to the Banff 97 classification.

-Category 2 includes antibody mediated changes which could be acute antibody-mediated rejection or chronic active antibody mediated rejection. The involvement of antibodies is proved by the detection of circulating anti-donor antibodies and the complement degradation product $\mathrm{C} 4 \mathrm{~d}$ -Category 3 includes borderline changes suspicious for acute T-cell mediated rejection (13). -Category 4 consists of T-cell mediated rejection which could be acute or chronic.

-Category 5 includes chronic changes of graft rejection and category 6 remains same as in the previous classification.

Minor modifications to Banff 2007 classification (table 3) were done in 2009 (Banff 2009 meeting report) and 2011 (Banff 2011 meeting report) as indicated below.

Banff 2009

C4d deposition without morphologic evidence of acute rejection was included under "indeterminate acute antibody mediated rejection" - Category 2. 
There were no changes to the scoring ( $t 1, \mathrm{t} 2, \mathrm{t} 3$, i1, i2, i3, v1, v2, v3) and grading categories.

\section{Banff 2011}

The 11th Banff meeting was held in 2011 with a focus on refining diagnostic criteria for antibody-mediated rejection (ABMR). The major outcome was the acknowledgement of

C4d (-) negative ABMR in kidney transplants. Banff classification remains unchanged.

A new Banff working group was created to define diagnostic criteria for $A B M R$ in renal transplants independent of $\mathrm{C} 4 \mathrm{~d}$. Results are expected to be presented at the 12th Banff meeting to be held in 2013 in Brazil.

\section{Antibody-mediated rejection and C4d staining}

The pioneering work by H. Feucht et al (14), more than a decade ago which led to the detection of the complement degradation product $\mathrm{C} 4 \mathrm{~d}$ in renal allograft biopsies contributed to major changes in the understanding and classification of kidney transplant pathology.

C4d is the degradation product of the activated complement factor $\mathrm{C} 4$, a component of

the classical complement cascade that is typically initiated by binding of antibodies to specific target molecules. There is covalent binding of the degradation product $\mathrm{C} 4 \mathrm{~d}$ to endothelial cell surfaces and extracellular matrix components of vascular basement membrane near the sites of $\mathrm{C} 4$ activation. $\mathrm{C} 4 \mathrm{~d}$ is also found in intracytoplasmic vacuoles of endothelial cells. Since C4d is only very rarely observed along peritubular capillaries in native kidneys, the accumulation of $\mathrm{C} 4 \mathrm{~d}$ along the walls of peritubular capillaries in the renal cortex and medulla is considered to be "transplant specific".

It has been reasonably concluded that the immunohistochemical detection of $\mathrm{C} 4 \mathrm{~d}$ in renal allografts is a "foot print" of an antibody response. In the majority of $\mathrm{C} 4 \mathrm{~d}$ positive allograft recipients, circulating donor specific anti-bodies against MHC class I or class II can be often found (15). C4d is scored in renal allograft biopsies based on immunohistochemical or immunofluorescence studies using either formalin-fixed and paraffin-embedded biopsy samples or alternatively fresh frozen tissue (Fig.5). 
However, formalin fixation and paraffin embedding results in decreased sensitivity to detect $\mathrm{C} 4 \mathrm{~d}$ and immunofluorescence studies (Fig.6) are regarded as the "gold standard" for identification of complement degradation product C4d. A, strong, diffuse and circumferential staining of peritubular capillaries in non-fibrotic and non-necrotic cortical and/or medullary

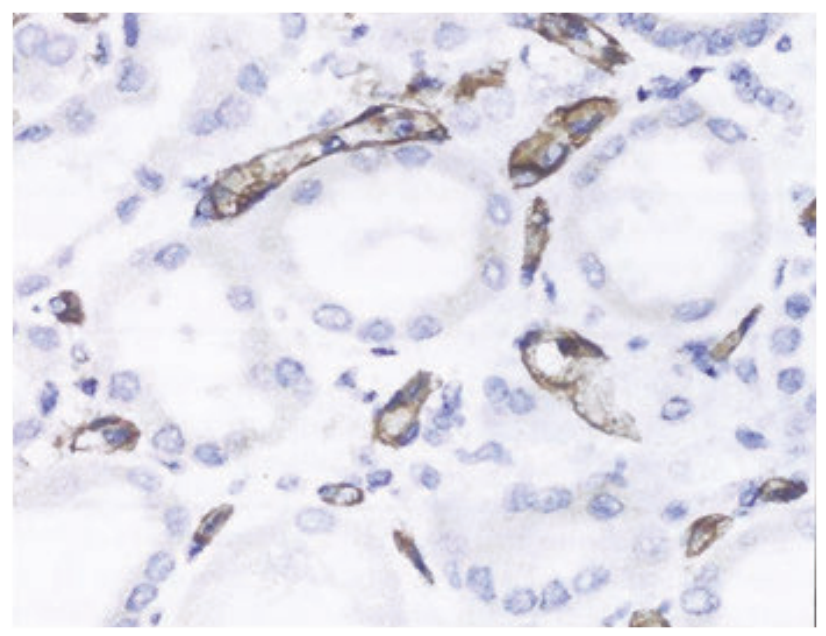

Fig 5: Staining for C4d along peritubular capillaries on formalin fixed and paraffin-embedded tissue sections (H \&E x 400). regions is generally considered to be diagnostic. C4d deposits in other locations, including vessels with arteriolosclerosis, atrophic tubular basement membranes, endothelial surfaces of large arteries and glomerular basement membrane are currently considered to be nondiagnostic and should not be used in the clinical decision making (16

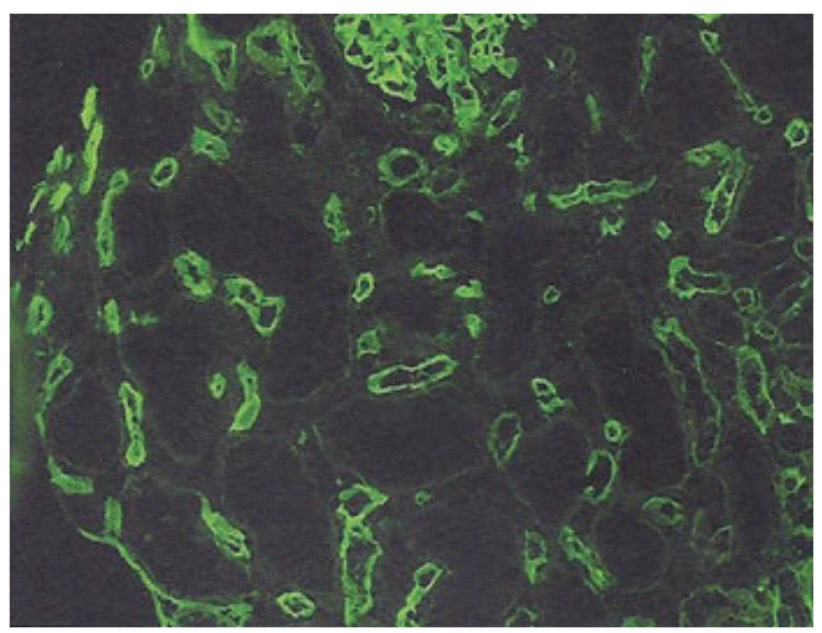

Fig 6: Immunofluorescence study with a monoclonal antibody directed against $\mathrm{C} 4 \mathrm{~d}$, on fresh frozen tissue (H \&E x 400).

Updated Banff classification scheme of renal allograft rejection has introduced a scoring scheme for C4d staining results (Table 4).

Table 4 Scoring of C4d staining (16)

\begin{tabular}{|l|l|l|}
\hline $\mathrm{C} 4 \mathrm{~d} 0$ & Negative & $0 \%$ \\
\hline $\mathrm{C} 4 \mathrm{~d} 1$ & Minimal C4d stain/detection & $1-10 \%$ \\
\hline $\mathrm{C} 4 \mathrm{~d} 2$ & Focal C4d stain/positive & $10-50 \%$ \\
\hline $\mathrm{C} 4 \mathrm{~d} 3$ & Diffuse C4d stain/positive & $>50 \%$ \\
\hline
\end{tabular}


Overview of the treatment modalities of renal allograft rejection

A hyperacute rejection manifests within minutes following transplantation and treatment is immediate removal of the tissue. Chronic rejection is generally considered irreversible and poorly amenable to treatment. Re-transplant is generally indicated if feasible. Acute rejection is treated with one or multiple of a few strategies which include administration of immunosuppressive drugs such as high dose corticosteroids, calcineurin inhibitors, azathioprine etc. Immunosuppressive therapy can be combined with antibody-based treatment such as monoclonal anti-IL-2R $\alpha$ receptor antibodies, anti T-cell antibodies or anti CD-20 antibodies (Rituximab).

\section{Summary}

Kidney transplantation which is considered as an effective way of treating patients with end stage renal disease, has achieved high standards over the last few years. Modern anti rejection drug regimens have substantially reduced rejection episodes and made long term survival possible. The pathology of allograft biopsies the fundamental backbone of all allograft related science, has provided crucial insights into the mechanisms of tissue injury and graft loss.

Histopathologists play an important role in identifying the different patterns of rejection in renal allograft biopsies and guiding the therapeutic management. Availability of ancillary techniques, helps the pathologist to render a definitive diagnosis which is more meaningful and clinically relevant, in terms of patient management.

However, in the rapidly advancing field of medical science, much more remains to be discovered in the future (17).

\section{References}

1. Mauiyyedi S, Colvin RB. Pathology of Kidney transplantation. In: Morris PJ (ed). Kidney Transplantation, 5th ed. W.B Saunders Co: Philadelphia, PA, 2001, pp 243-376.

2. Rosai J, Ackerman LV: Surgical Pathology. 9th edition. Philadelphia: Elsevier Inc. 2004.

3. Ruiz (ed) : Transplantation Pathology. New York : Cambridge University Press 2009.

4. Schwartz A, Gwinner W, Hiss M, Radermacher J, et al. Safety and adequacy of renal transplant protocol biopsies. American Journal of Transplantation 2005; 5: 1992-1996.

5. Furness PN, Philpott CM, Chorbadjian MT, Nicholson ML, et al. Protocol biopsy of the stable renal transplant: a multicenter study of methods and complication rates. Transplantation 2003; 76: 969-973.

6. Nickeleit V, Andreoni K. The classification and treatment of antibody mediated renal allograft injury: 
Where do we stand? Kidney International 2007; 71: 7

7. Bagnasco SM, Tsai W, Rahman MH, Kraus ES, et al.

CD-20 positive infiltrates in renal allograft biopsies with acute cellular rejection are not associated with worse graft survival.

American Journal of Transplantation 2007; \&: 19681973.

8. Nickeleit V, Zeiler M, Gudat F, Thiel G, et al. Histological characteristics of interstitial renal allograft rejection. Kidney and Blood Pressure Research 1998; 21: 230-232.

9. Mihatsch MJ, Nickeleit V, Gudat F. Morphological criteria of chronic renal allograft rejection. Transplant Practice 1999; 31:1295- 1297.

10.Solez K, Colvin RB, Racusen LC, Sis B, et al. Banff' 05 Meeting report: differential diagnosis of chronic allograft injury and elimination of chronic allograft nephropathy (“CAN"). American Journal of Transplantation 2007; 7: 518-526.

11.Racusen LC, Solez K, Colvin RB, Bonsib SM, et al. The Banff 97 working classification of renal allograft pathology. Kidney international 1999; 55: 713-723

12.Solez K, Colvin R, Racusen L, Hass M, et al. Banff 07 Classification of Renal Allograft Pathology: Updates and future directions. American Journal of Transplantation 2008; 8: 753- 760.

13.Saad R, Gritsch HA, Shapiro R, Jordan M, et al. Clinical significance of renal allograft biopsies with "Borderline changes" as defined in the Banff Scheme. Transplantation 1997;
14.Feucht HE, Schneeberger H, Hillebrand G, Burkhardt K, et al. Capillary deposition of C4d complement fragment and early renal graft loss. Kidney international 1993; 43: 1333-1338.

15.Nickeleit V, Mihatsch MJ. Kidney transplants, antibodies and rejection: is $\mathrm{C} 4 \mathrm{~d}$ a magic marker? Nephrology Dialysis and Transplant 2003; 18: 2232-2239.

16.Nickeleit V, Zeiler M, Gudat F, Thiel G, et al. Detection of the complement degradation product $\mathrm{C} 4 \mathrm{~d}$ in renal allografts: diagnostic and therapeutic implications. Journal of American Society of Nephrology

2002; 13: 242-251.

17.Al-Aly Z, Reddivari V, Moiz A, Balasubramanian G, et al. Renal allograft biopsies in the era of C4d staining: the need for change in the Banff classification system. Transplant International 2008; 21: 268-275. 